\title{
N91:-28117" \\ XeCl LASER PUMPED IODINE LASER USING t-C4F9I
}

554.36

$266 \geq 5$

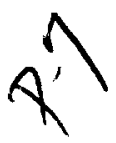

\author{
In H. Hwang and Kwang S. Han \\ Department of Physics \\ Hampton University \\ Hampton, VA
}

\begin{abstract}
An iodine photodissociation laser using $\mathrm{t}_{-} \mathrm{C}_{4} \mathrm{~F}_{9} \mathrm{I}$ as the active material was pumped by an $\mathrm{XeCl}$ laser. An iodine laser output energy of $3 \mathrm{~mJ}$ with pulse duration of $25 \mathrm{~ns}$ was obtained when the pumping pulse energy was $80 \mathrm{~mJ}$, the iodide pressure was $70 \mathrm{torr}$, and the reflectance of the output mirror was 85\%. The high pumping efficiency and low threshold pump power achieved in this experiment are attributable to the high absorption cross section at the pump laser wavelength $(308 \mathrm{~nm})$ of the iodide used.
\end{abstract}




\section{XeCl LASER PUMPED IODINE LASER USING t-C4F9I}

\section{Introduction}

Since the atomic iodine photodissociation laser was discovered by Kasper and Pimentel in 1964 [1], numerous research efforts have been concentrated on this laser to increase the power level needed for laser fusion $[2,3]$. The fusion-experiment-oriented iodine laser is constructed in a master-oscillatorpower-amplifier (MOPA) architecture [4].

The iodine laser is also being considered as a direct solar pumped laser for space applications such as the laser propulsion for an orbital transfer vehicle. The first solar simulator pumped iodine laser was reported in 1981 by Lee, et. al. [5]. This laser was operated in quasi-continuous-wave mode. After this report, various iodine compounds were tested and evaluated as candidates for direct solar pumped laser materials $[6,7,8]$.

Although many space applications are best served by a continuous wave (CW) laser, a continuously pulsed laser also has good applicability in space, especially for laser propulsion. If weak pumping (such as solar radiation pumping) of laser materials exists, use of a MOPA system is essential for obtaining high peak power when the laser material has a long upper-state lifetime like an iodine atom. In previous research, the feasibility of the oscillator-amplifier scheme was proposed and tested for the solar pumped iodine laser [7.9].

In order to be incorporated in the MOPA system, the iodine laser oscillator must generate temporally smooth and short pulses. In the fusion-oriented-iodine-laser-experiment the short pulse is provided by mode-locking of the flashlamp-pumped oscillator. However, the repetition rate of the flashlamp-pumped oscillator is usually very low $(<5 \mathrm{~Hz})$, and the mode-locking devices are generally complicated. An excimer laser pumped iodine laser oscillator was demonstrated by Fill et. al. [10]. In their experiment, a 500-mJ excimer laser was used for the pumping of the iodine laser, and a temporally smooth laser pulse with duration from $2.6 \mathrm{~ns}$ to $12 \mathrm{~ns}$ was obtained by using $\mathrm{i}-\mathrm{C}_{3} \mathrm{~F}_{7} \mathrm{I}$ as the laser material.

In this report, a XeCI laser pumped iodine laser using $\mathrm{t}_{-} \mathrm{C}_{4} \mathrm{~F}_{9} \mathrm{I}$ is described. This laser oscillator has much a higher efficiency and a lower threshold pump power than the system using $\mathrm{i}-\mathrm{C}_{3} \mathrm{~F}_{7} \mathrm{I}$. The laser output energy dependence on the gas-filled pressure and on the reflectance of the output mirrors are measured for both longitudinal and transverse pumping.

\section{XeCI Excimer Laser}

A laboratory built $\mathrm{XeCl}$ laser was used for the pumping of the iodine laser in this experiment. The electrical circuit of the $\mathrm{XeCI}$ laser is shown in Fig. 1 . A pyrex tube with an I.D. of $0.1 \mathrm{~m}$ and a length of $0.66 \mathrm{~m}$ was used as the laser chamber. The two brass electrodes were rounded in order not to develop an arc discharge in the discharge volume. The width of the electrodes was $20 \mathrm{~mm}$, and the length was about $0.45 \mathrm{~m}$. The separation between the two electrodes was $20 \mathrm{~mm}$. Seventeen pairs of arc arrays were located beside the electrodes so that the discharge volume could be preionized uniformly. The optimized gas composition was HCI: Xe: $\mathrm{Ar}: \mathrm{He}=0.2 \%: 5.9 \%: 19.5 \%: 74.4 \%$ at a total pressure of $2 \mathrm{~atm}$. The addition of argon gas improved the discharge uniformity at the higher operating voltage. 
When the XeCI laser was operated at the charging voltage of $25 \mathrm{kV}$, the output energy was $80 \mathrm{~mJ}$ per pulse. If the charging voltage was increased, the output energy also increased, but an arc discharge developed between the electrodes. Thus, operation above $25 \mathrm{kV}$ was not pursued. A typical laser pulse is shown in Fig. 2. The half width (FWHM) of the XeCI laser pulse was about $25 \mathrm{nsec}$, and no significant variation of the laser pulse was found for prolonged operation.

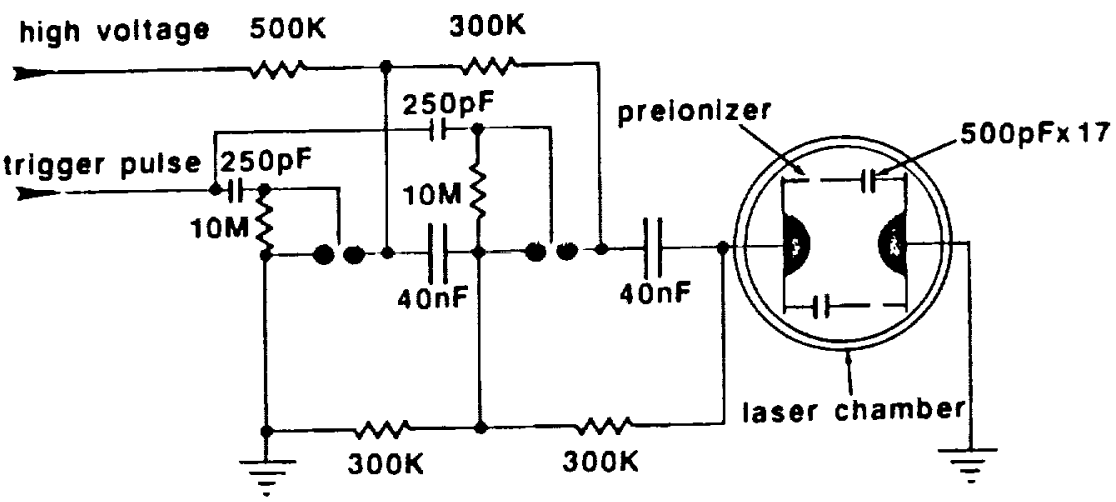

Figure 1

Electrical circuit diagram of the $\mathrm{XeCI}$ excimer laser.

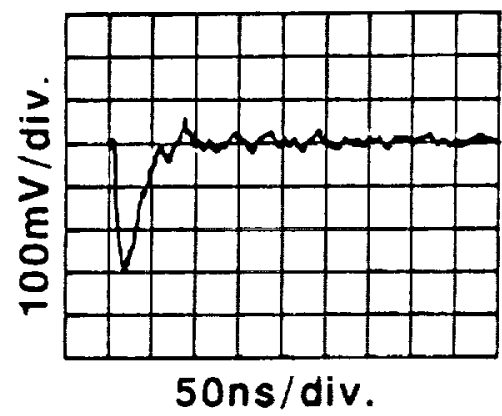

Figure 2

A typical pulse shape of the $\mathrm{XeCI}$ laser output. 


\section{Iodine Laser Experiment}

The iodide used in this experiment was $\mathrm{t}-\mathrm{C}_{4} \mathrm{~F}_{9} \mathrm{I}$ (perfluoro-tertiary-butyl iodide). This material was chosen mainly due to the absorption cross section at the wavelength of $\mathrm{XeCI}$ laser light $(308 \mathrm{~nm})$. The published data show that the absorption cross section of $t-C_{4} F_{9} I$ at $308 \mathrm{~nm}$ is about $3.6 \times 10-10 \mathrm{~cm}^{2}$ [7]. Also, $t-C_{4} F_{9} I$ has shown good chemical reversibility in a flashlamp-pumped system $[6,7]$, and thus the iodine molecule buildup in the laser cell is minimized. The main disadvantage of this iodide is the low vapor pressure ( $\approx 85$ torr) at room temperature. The other chemical kinetic properties are nearly the same or better than other commonly used perfluoralkyl iodides in the flashlamp-pumped experiment [7]. The quantum yield of excited atomic iodine in the UV photodissociation is nearly unity [11].

A quartz cuvette with a square cross section of $1 \mathrm{~cm}^{2}$ and length of $50 \mathrm{~mm}$ was used as the iodine laser cell. The windows of the laser cell were nearly perpendicular to the optic axis of the laser cell. Both longitudinal and transverse pumping of the iodine laser were employed.

When the iodine laser was pumped longitudinally, a dichroic mirror was used to introduce the $\mathrm{XeCI}$ laser light into the iodine laser cell. The dichroic mirror transmitted about $80 \%$ of the XeCI laser light and fully reflected the iodine laser light. The XeCI laser light was directed through the dichroic mirror and was focused into the center of the laser cell by a quartz spherical lens of focal length $0.2 \mathrm{~m}$. When the iodine laser was pumped transversely, a quartz cylindrical lens of focal length $0.3 \mathrm{~m}$ was used to focus the $\mathrm{XeCI}$ laser light into the iodine laser cell.

The output energy dependence on the gas filled pressure at different output mirror reflectances is shown in Figs. 3 and 4. As shown in Fig. 3, there is an optimum iodide pressure for each output mirror reflectance for the longitudinal pumping. However, for the transverse pumping, the laser out put energy increases monotonically with the $\mathrm{t}-\mathrm{C}_{4} \mathrm{~F}_{9} \mathrm{I}$ pressure as shown in Fig. 4 The results in Figs. 3 and 4 were taken when the laser was operated at $2-\mathrm{Hz}$ repetition rate. When the pulse repetition rate was increased to $5 \mathrm{~Hz}$, the laser output energy per pulse was reduced slightly $(\approx 10 \%)$ due to the reduction of the $\mathrm{XeCI}$ laser pumped energy.

A typical laser output is shown in Fig. 5. The half width (FWHM) of the pulse is about 25 nsec. The iodine laser onset is delayed from the XeCI pump laser and occurs at the end of the pump pulse. The pulse shape is nearly the same for both pumping geometries.

\section{Discussion}

In this experiment, a XeCI laser pumped iodine laser was developed and tested by using $\mathrm{t}-\mathrm{C}_{4} \mathrm{~F}_{9} \mathrm{I}$ as the laser material. This lasant $t-C_{4} F_{9} I$ has a larger absorption cross section at the XeCI laser line compared with other iodides used in the laser experiment, such as $\mathrm{i}-\mathrm{C}_{3} \mathrm{~F}_{7} \mathrm{I}$. The larger absorption cross section allows the high utilization of pumping energy in short gain length and low pressure operation of the iodine laser. The low-pressure operation of the iodine laser is suitable for single longitudinal mode output because of the reduced pressure broadening of the gain profile. The single-longitudinal mode operation of the laser oscillator is necessary to obtain a temporally smooth pulse.

The iodine laser described in this report was operated up to $5 \mathrm{~Hz}$, which was limited by the power supply of the XeCI laser. Further increase of the repetition rate can be made by scaling up the pump laser system. The iodine laser was operated in a sealed-off mode. There was no significant reduction of the laser output energy after a few hundred pumpings with a single fill $t-\mathrm{C}_{4} \mathrm{~F}_{9} \mathrm{I}$ in the laser cell. There 
was also no noticeable change in the $\mathrm{t}-\mathrm{C}_{4} \mathrm{~F}_{9} \mathrm{I}$ gas after a few hundred pumpings and no deposits on the wall of the laser cell (contrary to the report of Ref. 10 where $t-C_{3} F_{7} I$ was used). This may be attributable to the superior chemical reversibility of the iodide used.

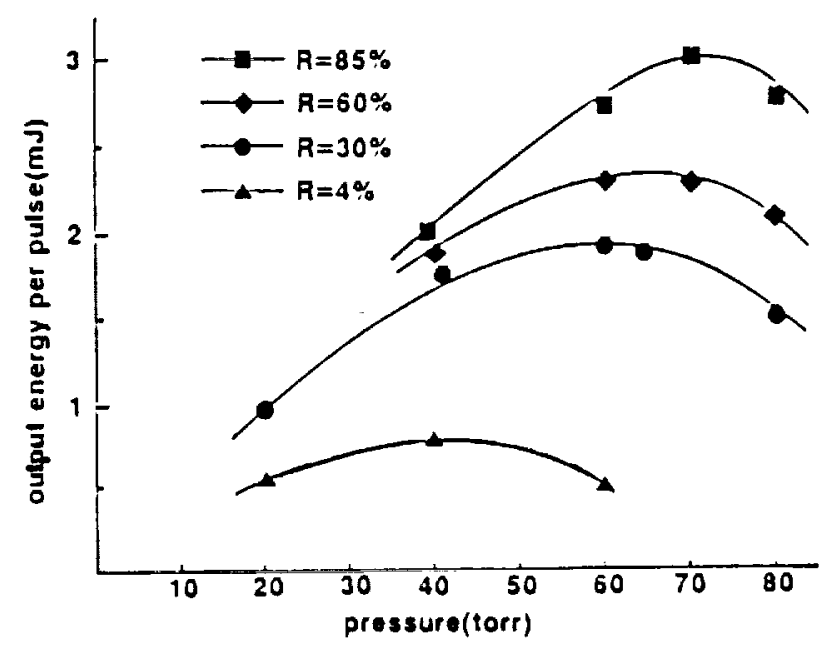

Figure 3 - Iodine output energy dependence on the gas fill pressure in the laser cell at each output mirror reflectance. The iodine laser was pumped longitudinally with the XeCI laser of pulse energy $80 \mathrm{~mJ}$.

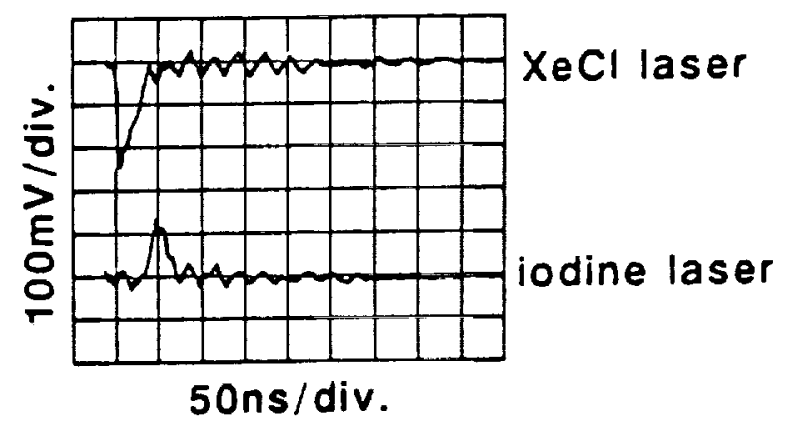

Figure 4 - Iodine laser output energy dependence on the gas fill pressure when the iodine laser was pumped transversely. The pumping energy from the XeCI laser was fixed to $80 \mathrm{~mJ}$. 


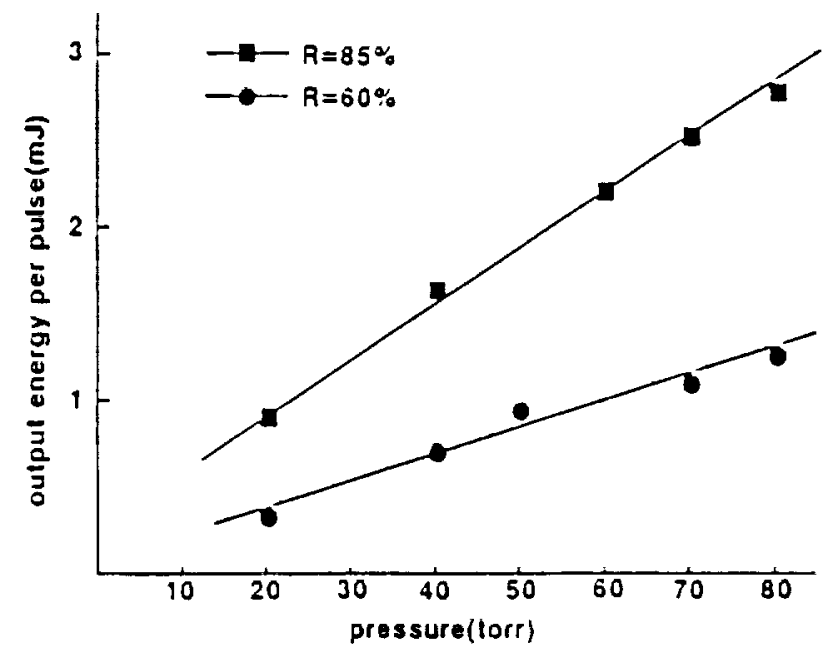

Figure 5 - A typical iodine laser pulse shape which is compared with the pumping pulse. (a) XeCI pump laser pulse. (b) Iodine laser output pulse.

\section{Conclusion}

An iodine laser oscillator pumped by an $\mathrm{XeCI}$ laser was developed using $\mathrm{t}-\mathrm{C}_{4} \mathrm{~F}_{9} \mathrm{I}$ as the laser material, and a $3 \mathrm{~mJ}$ laser output energy was obtained with only $80 \mathrm{~mJ}$ pumping energy. Compared with previous results, the pumping energy was dramatically reduced in this experiment, The pumping efficiency (i.e., the ratio of the iodine laser energy and the $\mathrm{XeCI}$ laser energy) was $3.75 \%$. This experiment also demonstrated a repetitive operation of the iodine laser oscillator with a stable output. Since the threshold pumping energy $(20 \mathrm{~mJ})$ is low for $\mathrm{t}-\mathrm{C}_{4} \mathrm{~F}_{9} \mathrm{I}$, a moderate size XeCI laser may suffice for the pumping of the high-repetition-rate laser oscillator required for solar pumped master-oscillator power-amplifier systems.

\section{Acknowledgments}

This work is supported by NASA grant NAG1-441.

\section{References}

1. J.V.V. Kasper and G.C. Pimentel, Appl. Phys. Lett. 5, 231 (1964).

2. G. Brederlow, E. Fill and K.J. Witte, "High-Power Iodine Laser," Springer-Verlag, New York, N.Y. (1983). 
3. S.B. Kormer, Izvesteriya Akademii Nauk SSSR. Seriya Fizicheskaya 44, 2002 (1980).

4. K.J. Witte, Czech. J. Phys. B34, 790 (1984).

5. J.H. Lee and W.R. Weaver, Appl. Phys. Lett. 39, 137 (1981).

6. J.H. Lee, J.W. Wilson, T. Anderson, D.H. Hume, W.R. Weaver and B.M. Tabibi, Optics Comm. 53, 367 (9185).

7. B.M. Tabibi, M.H. Lee, J.H. Lee and W.R. Weaver, Proceedings of International Conference on Lasers' 86, pp 144-149 (1986).

8. R.J. Deyoung, IEEE. J. Quant. Electron. OE-22, 1019 (1986).

9. I.H. Hwang, J.H. Lee and M.H. Lee, Optics Comm. 58, 47 (1986).

10. E. Fill, W. Skrlac and K.J.K. Witte, Optics Comm. 37, 123 (1981).

11. A.B. Alekseev, A.M. Pravilov, I.I. Didorov and V.A. Skorohodov, Sov. J. Quantum Electron. 17, 1539 (1987). 